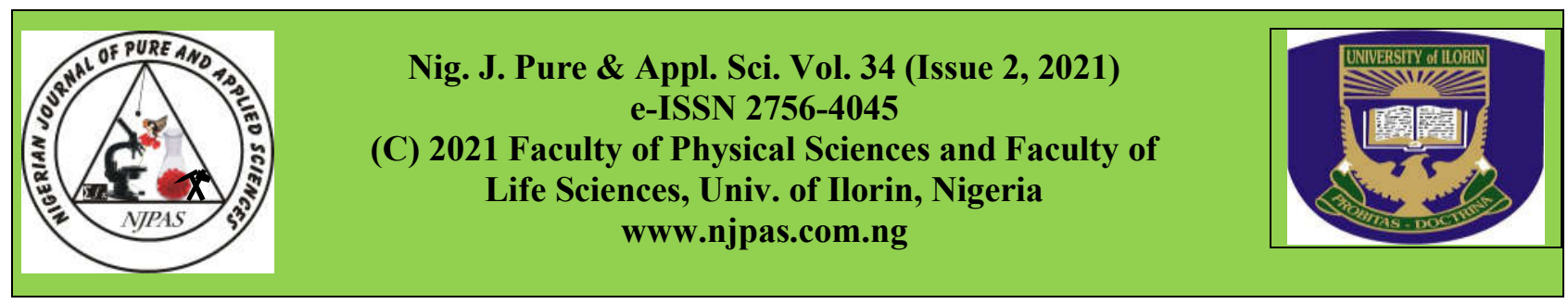

\title{
Productivity of Cowpea (Vigna unguiculata (L) Walp) genotypes under Varying Population Densities
}

\author{
*Afolabi, Michael Segun, Ojeleye Abiola Elizabeth, Olalekan Kazeem Kolapo and Shittu Kabiru Alani
}

Page | 4089

Department of Agronomy, Osun State University, Ejigbo Campus, Osun State

Date Received: 27-04-2021

Date Accepted: 28-08-2021

DOI: https://doi.org/10.48198/NJPAS/21.xxx

\begin{abstract}
To investigate the influence of three population densities $(88,000,44,000$, and 29,000 plants/ha) on the growth and yield of five cowpea varieties (IT97K-461, IT97K-568-18, IT98K-131-2, IT99K-1060 and IT99K-245), a field experiment was conducted at Teaching and Research farm of Landmark University. Growth parameters such as germination count, plant height, and number of trifoliate leaves per plant, number of branches per plant, flower production dynamics and dry matter determination were measured, while harvest index, shelling percentage, number of seeds per pod, see weight pods per plant and grain yield were measured at harvest as yield components. Data were subjected to statistical analysis of variance (ANOVA) using SAS Institute. Significant means were separated using Duncans Multiple Range Test at 5\% probability level. Results revealed that there were no constant trends in growth parameters response to population density. Plant height and flower production decreased with increased population density, while number of leaves per plant and dry matter increased with increasing population density. There was no appreciable effect of population density on branching. All these parameters were significantly $(\mathrm{p} \leq 0.05)$ affected with variety, while there was no significant $(\mathrm{p} \leq 0.05)$ interaction effect. Most yield components decreased with increasing population density while grain yield however, slightly increased with increasing population density. All yield parameter and grain yield significantly varied with variety, while there were no appreciable interaction effect. In conclusion, a population density of 88,000 plants /ha gives the highest growth and grain yield. While, variety, IT97-568-18 gives the higher growth and grain yield than other varieties studied.
\end{abstract}

Keywords: Population density, Shelling percentage, flower production dynamics

\section{Introduction}

Cowpea (Vigna unguiculata (L) Walp) is one of the increasingly cultivated grain legumes of the tropical and subtropical countries. Cowpea is the most important source of protein for human being and livestock (Ajeigbe et al., 2010). Cowpea can be used as a primary source of protein for Africans who cannot afford animal protein (Brader, 2002).
In most areas they are grown and eat in various ways either alone or mixed with maize, rice, fish or "gaari" which supplies $40 \%$ of the daily protein. Flour is made into fried or boiled cakes as 'akara' or 'moinmoin'. Despite the importance of this crop, however, it has been observed that the yield of cowpea in southern guinea savannah of Nigeria has been lower than world average which ranges

Corresponding Author: Michael Segun Afolabi

Department of Agronomy, Osun State University, Ejigbo Campus, Osun State

Email: afolabimike97@gmail.com 
between $200-600 \mathrm{~kg} / \mathrm{ha}$. A lot of factors may be responsible for this under production. Ajeigbe et al. (2006) stated that factors responsible for low yield production include pest infestation, disease and poor management practices. Low yield in

Page | 4090 cowpea production can also be attributed partly due to inefficient partitioning of dry-matter to the seeds and varietal factors.

A population density of less than 33,000 plants per hectare has been reported from farmers farm (Dugje et al., 2009). At this density, the crop does not cover the ground completely, thus allowing the weeds to grow and reducing the efficiency of land utilization. Experiment has reported that cowpea responded very well to inter row spacing of $60 \times 30$ $\mathrm{cm}$ giving a plant population of 55,000 plant per hectare (Kamara et al., 2014). In Nigeria, Anonymous, (2008 showed that a population of 87,000 plants per hectare was ideal for erect and semi erect type. At low densities, competition may not occur at all and resources are not efficiently used. It is therefore the objectives of this present study is to investigate the influence of inter row spacing on growth and yield of cowpea varieties in southern guinea savannah of Nigeria and also to determine if cowpea response to increasing density is genotype dependent.

\section{Materials and Methods}

Field experiment was conducted at the Teaching and Research ( $T$ and $R$ ) farm of Landmark University Omu Aran, Kwara State (Latitude 80 $28^{1} \mathrm{~N}$, Longitude $4034^{1} \mathrm{E}$ and annual rainfall of $1005 \mathrm{~mm})$ in the southern Guinea savannah of Nigeria (Afolabi et al., 2020). Composite soil samples were collected randomly from the trial site at the depth of $0-15 \mathrm{~cm}$ with soil auger prior to planting. The samples were analysed in the Soil, Water and Material Testing Laboratory of Lower Niger River Basin Development Authority, Ilorin, Nigeria for physico-chemical analysis (Table 1). Collected samples were air-dried and passed through $2 \mathrm{~mm}$ sieve The sieved samples were analyzed for chemical properties. Soil $\mathrm{pH}$ was determined with a glass electrode $\mathrm{pH}$ meter in distilled water using 1:1 soil: water as described by Thomas (1996). Total nitrogen was determined by the macro-Kjeldahl method (Bremner, 1996); Exchangeable cations $\left(\mathrm{Ca}^{2+}, \mathrm{Mg}^{2+}, \mathrm{K}^{+}\right.$and $\left.\mathrm{Na}^{+}\right)$ were extracted with neutral $1.0 \quad \mathrm{M} \mathrm{NH} \mathrm{NH}_{4} \mathrm{OA}_{\mathrm{C}}$ solution of. The $\mathrm{K}^{+}, \mathrm{Ca}^{2+}$ and $\mathrm{Na}^{+}$concentrations in the extract were determined using the flame photometer while $\mathrm{Mg}^{2+}$ was determined using the atomic absorption spectrophotometer (AAS). For the determination of soil organic carbon, the Walkley-Black wet oxidation method (Nelson and Sommers, 1982) was adopted, Available P by the Bray-1 method as described by Kuo (1996) For soil physical properties, particle size analysis was determined by hydrometer method according to Okalebo et al., (2002). Soils of the sites for the two locations are sandy clay loam, low in nitrogen and other micro elements (Table 1).

The experiment was laid out as a Randomized Complete Block in split plot arrangement with the population consisting of the population density as main block and varieties as subplot. The population density were achieved by planting at inter-row spacing of $30 \mathrm{~cm}, 60 \mathrm{~cm}$ and $90 \mathrm{~cm}$ equivalent to $88,000,44,000$ and 29,000 plants per hectare respectively. The plot size is $15 \mathrm{~m}^{2}$ consisting 2 rows of $5 \mathrm{~m}$ long. The varieties considered were IT97K-461-4, IT97K-568-18, IT98K-131-2, IT99K-1060 and IT99K-1245 which were collected from the International Institute for Tropical Agriculture (IITA) Ibadan. Land preparation was carried mechanically using tractor to plough. Ridging was done at 2 weeks after to allow proper decaying of debris. Direct sowing was done at two seeds per hole in double row on a ridge. Weeds were controlled twice manually with hoe, first at 2 weeks after planting and secondly at 4-5 weeks after planting to ensure a clean field which is the most common method used by farmers in cowpea production. Weeding was at 6 weeks after sowing (WAS), all plot were sprayed with insecticide landacyhalothrine $25 \mathrm{EC}$ at a rate of $2 \mathrm{ml}$ per litre. The spraying continued at interval until pod 
maturity. Data collection started at 2WAS and the following parameters were recorded

\section{Growth parameters}

Five plants were tagged randomly in each plot for Page | 4091 ease growth parameter collection according to Watson et al., (2002). Growth parameters recorded are: Plant height (measurement was from the soil surface to the last foliage leaf using measuring tape in centimetre); Number of trifoliate leaves; Number of branches, and Numbers of flowers.

Proximate data taken were; Dry matter determination which were taken at the commencement of flowering using three samples in a plot which were randomly uprooted according to Vogl et al., (2004). The root was cut from stem, packed separately and oven dried at $100^{\circ} \mathrm{c}$ for 48 hours.

Yield Parameters taken at harvest, harvesting was done at $11 \mathrm{WAP}$ when all pods had been dried; data were taken on;

$$
\text { Number of pod per plant }=\frac{\text { Total number of pods }}{\text { Number of tagged plants }}
$$

Number of seeds per pod, Seed weight, Pod weight determination, Grain weight, Stoffer weight, Shelling percentage: The shelling percentage from each plot was recorded using the expression below:

$$
\text { Shelling percentage }(\mathrm{SP})=\frac{\text { Grain weight } \times 100}{\text { Pod weight }}
$$

Harvest index: the ration of economic yield to that of total biomass is harvest index. Biomass is stoffer weight plus pod weight

$$
\text { Harvest index }=\quad \frac{\text { Economic yield }}{\text { Total biomass }}
$$

\section{Data analysis}

All data collected data were subjected to statistical analysis of variance (ANOVA) using SAS 9.4 (2013). Significant means were separated using Duncans Multiple Range Test at 5\% probability level.

\section{Results}

\section{Effects of plant growth parameters}

The result of the analysis of variance for germination shows that both the main effect of population density and variety as well as their interaction were significant (Table 2). The table also show that the effect of population density, variety and the population $\mathrm{x}$ variety interaction were generally significant for plant height throughout the measuring periods except at 2 and 3 WAP for population density. Table 3 showed that germination was significantly higher at density of 44,000 plants per hectare, while 88,000 plants per hectare and 29,000 plants per hectare showed similar germination. IT97K-461-4 has significantly lower germination than other varieties. IT98K-1312, IT99K-1060 and IT99K-1245 have similar germination which was significantly higher in germination than IT97K-568-18. However, significant interaction effect revealed that while population density had no effect in germination of varieties IT97K-568-18, IT99K-1060 and IT99K1245.

Germination in variety IT97K-461-4 and IT98K131-2 was significantly reduced by the lowest population density (Table 4). Plant height decreased with increased population density with the plant at the highest population density showing significantly lower height than in the other two population densities. Plant height was highest at population density of 29,000 , the values however, were not significantly taller than in plans at 44,000 plants per hectare. Plant height was significantly highest with variety IT97K-568-18 and significantly lowest with variety IT97K-461-4 throughout the measurement period (Table 3). Analysis of variance for number of trifoliate leaves show that the effects of population density were significant from 4 WAS to 7 WAS, while the effects of variety were significant throughout the measurement period except at 2 WAS. Population density $\mathrm{x}$ variety effect were also significant 
throughout the period except at 2 WAS and 5 WAS (Table 5).

Table 6 shows that number of leaves increases with increase in days of planting. Number of trifoliate leaves increased with increasing population density throughout the measurement period with leaves production at population density 88,000 plants/ha significantly higher than at both 44,000 and 29,000 plants/ha which showed similar leave production. IT97K-568-18 and IT99K-1245 gave significantly $(p \leq 0.05)$ higher number of leaves more than in the other varieties throughout the measurement period. The result of analysis of variance for branches (Table 7) shows that both the effects of population density and variety as well as their interaction were not significant $(p \leq 0.05)$ throughout the period of data collection with the exception of 4 WAP for variety and interaction. Table 8 show that branches initially increase with increase in days of planting until a constant number is recorded at 4WAP till 7 WAP. Numbers of branches were neither affected by population density nor by variety. There was also no significant effect.

\section{Effect on dry matter production}

Dry matter was not significantly $(\mathrm{p} \leq 0.05)$ affected by both density and density by variety interaction, while it is significantly $(\mathrm{p} \leq 0.05)$ influenced by the variety (Table 9)

Table 10 show that dry matter was higher with population density of 88,000 plants/ha and least with 44,000 plants /ha. However, the differences were not significant. IT97K-568-18 has the highest dry matter which was significantly $(\mathrm{p} \leq 0.05)$ higher from those of other varieties. IT98K-131-2 was significantly lower from other varieties.

\section{Effects of flower production dynamics}

Analysis of variance for flower production show that that effect of population density were generally not significant throughout the period of flower counting with the exception of days 3 and 4 . However, the effects of variety were generally significant with the exception of days 3 and 4 . However, the effect of variety was generally significant with the exception of days 7.8.9 and 15. Density $x$ variety effect was only significant on few occasions during measurement (Table 11). Flower production increased with decrease in population density with production at the highest population density significantly lower than in the two lower densities which showed similar flower production from day 1 to day 7 . Thereafter flower production was similar for all the three varieties. IT97K-461-4 and significantly lowest with variety IT98K131-2 throughout the measurement period (Table 12).

\section{Effect on yield components}

Analysis of variance for yield and yield component shows that the effect of population except seeds per pod. Similarly, the effects of variety were only significant $(\mathrm{p} \leq 0.05)$ for number of seeds per pod and seed weight, (Table 13), while the interaction effect of density $x$ variety were only significant for shelling percentage and number of pods per plant. Table 14, showed that harvest index was highest at $29,000 \mathrm{plants} / \mathrm{ha}$ and least at 44,000 plants/ha, even though the IT98K-131-2 and IT99K-1245 had similar harvest index which was significantly $(p \leq 0.05)$ higher than those of IT97K-461_4 and IT99K-1060.

Shelling percentage was not significantly different with all density even though it increased with increasing density. IT99K-125 showed significantly $(\mathrm{p} \leq 0.05)$ higher shelling percentage than other varieties.

Number of seeds was highest at 29,000 plants/ha although this was not significantly $(\mathrm{p} \leq 0.05)$ higher than 44,000 plants /ha. Number of seeds per pod decreased with increasing density. IT97K-568-18 has significantly higher number of seeds per pod than the other varieties. Number of seeds per pod

was significantly $(\mathrm{p} \leq 0.05)$ lowest in IT97K-461, although the value was not significantly lower than in IT99K-1245. 
Pod per plant decrease with increased population with significant $(\mathrm{p} \leq 0.05)$ difference between 88,000 pants /ha and 29,000 plants/ha. Number of seeds per plant at 44,000 plants/ha was neither significantly lower than in 29,000 plants/ha higher Page | 4093 than 88,000 plants/ha. IT97K-461 and IT99K-1060 was not significantly different but significantly higher than other varieties.

Seed weight shows no significant $(\mathrm{p} \leq 0.05)$ difference. IT97K-461-4 has the highest seed weight and value was significantly higher than in other varieties which showed similar seed weight.

Grain yield slightly increased with increasing density even though the differences were significant. IT97K-568-18, IT98K-131-2, IT99K1245 and IT99K-1060 had significantly $(\mathrm{p} \leq 0.05)$ higher grain yield which was however not significantly lower in IT99K-1060.

Table 1: Physical and Chemical Properties of Soil in the Experimental Sites

\begin{tabular}{ll}
\hline Properties & Value \\
\hline Sand \% & 73.00 \\
Clay \% & 6.00 \\
Silt \% & 21.00 \\
Textural class & Sandy clay loam \\
pH $\left(\mathrm{H}_{2} 0\right)$ & 5.70 \\
Organic matter \% & 1.70 \\
Nitrogen \% & 0.07 \\
Phosphorus $(\mathrm{mg} / \mathrm{kg})$ & 8.80 \\
$\mathrm{Ca}^{2+}(\mathrm{cmol} / \mathrm{kg})$ & 1.72 \\
\hline
\end{tabular}

Table 2: Mean square from the analysis of variance for germination and plant height

\begin{tabular}{lclllllll}
\hline SV & Df & Germ & 2 WAS & 3 WAS & 4 WAS & 5 WAS & 6 WAS & 7 WAS \\
\hline Density & 2 & $424.09^{*}$ & $0.34 \mathrm{~ns}$ & $2.06 \mathrm{~ns}$ & $13.36^{* *}$ & $436.99^{*}$ & $1692.57^{* *}$ & $3158.77^{*}$ \\
Error (a) & 4 & 49.62 & 1.61 & 1.64 & 0.59 & 34.32 & 95.67 & 49.62 \\
Variety & 4 & $477.37^{* * *}$ & 6.48 & 7.79 & $9.93^{* * *}$ & $531.10^{* * *}$ & $1171.16^{* * *}$ & $477.37^{* *}$ \\
$\begin{array}{l}\text { Density } \\
\text { variety }\end{array}$ & $\mathrm{x}$ & $198.70^{* *}$ & $2.01^{* *}$ & 1.34 & $3.07^{* * *}$ & $130.30^{* * *}$ & $204.30^{* *}$ & $198.70^{* *}$ \\
Error (b) & 24 & 61.71 & 0.46 & 0.54 & 0.5151 & 24.18 & 57.20 & 61.71
\end{tabular}

$*, * *, * * *$ Significant $\mathrm{F}$ test at $0.05,0.01$ and 0.001 levels of probability respectively, "ns" not significant 
Table 3: Effect of population density and variety in germination and plant height

\begin{tabular}{|c|c|c|c|c|c|c|c|c|}
\hline & Treatment & Germination & $2 \mathrm{WAS}$ & $3 \mathrm{WAS}$ & $4 \mathrm{WAS}$ & $5 \mathrm{WAS}$ & $6 \mathrm{WAS}$ & $7 \mathrm{WAS}$ \\
\hline & 88,000 & $77.9^{\mathrm{b}}$ & $9.65^{\mathrm{a}}$ & $10.90^{\mathrm{b}}$ & $12.99^{\mathrm{b}}$ & $21.37^{\mathrm{b}}$ & $24.40^{\mathrm{b}}$ & $26.45^{\mathrm{c}}$ \\
\hline & 44,000 & $84.4^{\mathrm{a}}$ & $9.95^{\mathrm{a}}$ & $11.68^{\mathrm{a}}$ & $14.43^{\mathrm{a}}$ & $31.31^{\mathrm{a}}$ & $39.10^{\mathrm{a}}$ & $50.15^{\mathrm{b}}$ \\
\hline \multirow[t]{9}{*}{ Page | 4094} & 29,000 & $73.9^{\mathrm{b}}$ & $9.79^{\mathrm{a}}$ & $11.39^{\mathrm{ab}}$ & $14.77^{\mathrm{a}}$ & $30.00^{\mathrm{a}}$ & $45.00^{\mathrm{a}}$ & $52.80^{\mathrm{a}}$ \\
\hline & Sed & 2.57 & 0.46 & 0.47 & 0.28 & 2.14 & 3.57 & 0.73 \\
\hline & Variety & & & & & & & \\
\hline & IT97K-461-4 & $57.1^{\mathrm{c}}$ & $9.68^{\mathrm{b}}$ & $11.36^{\mathrm{b}}$ & $14.44^{\mathrm{b}}$ & $21.32^{\mathrm{c}}$ & $25.30^{c}$ & $28.00^{\mathrm{c}}$ \\
\hline & IT97-568-18 & $76.9^{\mathrm{b}}$ & $10.27^{\mathrm{a}}$ & $11.56^{\mathrm{b}}$ & $14.16^{\mathrm{b}}$ & $29.60^{\mathrm{a}}$ & $51.70^{\mathrm{a}}$ & $28.57^{\mathrm{a}}$ \\
\hline & IT98K-131-2 & $85.1^{\mathrm{a}}$ & $9.76^{\mathrm{b}}$ & $11.56^{\mathrm{b}}$ & $14.28^{\mathrm{b}}$ & $30.68^{b}$ & $44.50^{\mathrm{b}}$ & $50.36^{\mathrm{b}}$ \\
\hline & IT99K-1060 & $86.6^{\mathrm{a}}$ & $8.5^{\mathrm{c}}$ & $9.77^{\mathrm{c}}$ & $12.31^{\mathrm{c}}$ & $21.94^{\mathrm{c}}$ & $27.70^{c}$ & $32.19^{\mathrm{d}}$ \\
\hline & IT99K-1245 & $87.9^{\mathrm{a}}$ & $10.78^{\mathrm{a}}$ & $12.29^{\mathrm{a}}$ & $15.13^{\mathrm{a}}$ & $24.26^{\mathrm{c}}$ & $31.60^{\mathrm{c}}$ & $36.56^{\mathrm{c}}$ \\
\hline & Sed & 3.70 & 0.32 & 0.35 & 0.33 & 2.32 & 3.57 & 1.39 \\
\hline
\end{tabular}

*Mean values with the same letter along the same row are not significantly different, $\mathrm{p} \leq 0.05$

Table 4: Interactive effects of population and variety on germination

\begin{tabular}{lllll}
\hline Variety & 88,000 & 44,000 & 29,000 & Variety means \\
\hline IT97K-461-4 & 61.7 & 72.0 & 37.7 & 57.1 \\
IT97-568-18 & 69.7 & 80.7 & 80.3 & 76.9 \\
IT98K-131-2 & 86.0 & 88.3 & 81.0 & 85.1 \\
IT99K-1060 & 87.0 & 83.7 & 87.0 & 86.6 \\
IT99K-1245 & 85.0 & 95.3 & 83.3 & 87.9 \\
Population means & 77.9 & 84.4 & 73.9 & \\
\hline
\end{tabular}

s.e.d. for population $=2.57$; s.e.d. for variety $=3.7$; s.e.d. for population $\mathrm{x}$ variety $=6.29$

Table 5: Means square from the analyses of variance for number of trifoliate leave per plant

\begin{tabular}{|c|c|c|c|c|c|c|c|}
\hline \multirow[b]{2}{*}{ SV } & \multicolumn{7}{|c|}{ Plant height (weeks after planting (WAS) } \\
\hline & df & $2 \mathrm{WAS}$ & 3 WAS & $4 \mathrm{WAS}$ & 5 WAS & $6 \mathrm{WAS}$ & 7 WAS \\
\hline Density & 2 & $0.022 \mathrm{~ns}$ & $7.27 \mathrm{~ns}$ & $37.96^{*}$ & $90.82 * * *$ & $190.16^{* *}$ & $224.47 * * *$ \\
\hline Error (a) & 4 & 0.01 & 2.37 & 4.49 & 0.79 & 6.92 & 2.56 \\
\hline Variety & 4 & $0.08 \mathrm{~ns}$ & 23.61 & $17.80 * * *$ & 34.03 & $55.19 * * *$ & $61.42 * * *$ \\
\hline Density $\mathrm{x}$ variety & 8 & $0.08 \mathrm{~ns}$ & $3.04 * * *$ & $5.90 * *$ & 3.52 & $15.91 * *$ & $19.19^{*}$ \\
\hline Error (b) & 24 & 0.10 & 0.5 & 1.75 & 3.24 & 4.86 & 7.00 \\
\hline
\end{tabular}


Table 6: Effect of population density and variety on number of trifoliate leaves per plant

\begin{tabular}{lllllll}
\hline \multicolumn{7}{c}{ Plant height (weeks after planting (WAS) } \\
Treatment & 2 WAS & 3 WAS & 4 WAS & 5 WAS & 6 WAS & 7 WAS \\
\hline 88,000 & $2.07^{\mathrm{a}}$ & $6.47^{\mathrm{a}}$ & $13.53^{\mathrm{a}}$ & $23.47^{\mathrm{a}}$ & $29.80^{\mathrm{a}}$ & $30.67^{\mathrm{a}}$ \\
44,000 & $2.13^{\mathrm{a}}$ & $5.33^{\mathrm{b}}$ & $11.27^{\mathrm{b}}$ & $20.40^{\mathrm{b}}$ & $23.67^{\mathrm{b}}$ & $24.73^{\mathrm{b}}$ \\
29,000 & $2.07^{\mathrm{a}}$ & $5.20^{\mathrm{b}}$ & $10.47^{\mathrm{b}}$ & $18.60^{\mathrm{c}}$ & $23.60^{\mathrm{b}}$ & $23.40^{\mathrm{b}}$ \\
Sed & 0.11 & 0.56 & 0.78 & 0.32 & 0.96 & 0.59 \\
Variety & & & & & & \\
IT97K-461-4 & $2.11^{\mathrm{a}}$ & $8.56^{\mathrm{a}}$ & $13.56^{\mathrm{a}}$ & $22.67^{\mathrm{a}}$ & $27.33^{\mathrm{a}}$ & $28.11^{\mathrm{a}}$ \\
IT97-568-18 & $2.00^{\mathrm{a}}$ & $5.11^{\mathrm{b}}$ & $11.67^{\mathrm{bc}}$ & $21.33^{\mathrm{a}}$ & $25.22^{\mathrm{b}}$ & $5.78^{\mathrm{b}}$ \\
IT98K-131-2 & $2.22^{\mathrm{a}}$ & $4.89^{\mathrm{b}}$ & $10.00^{\mathrm{d}}$ & $22.56^{\mathrm{a}}$ & $26.89^{\mathrm{a}}$ & $26.56^{\mathrm{a}}$ \\
IT99K-1060 & $2.11^{\mathrm{a}}$ & $5.00^{\mathrm{b}}$ & $12.67^{\mathrm{ab}}$ & $19.00^{\mathrm{b}}$ & $21.56^{\mathrm{c}}$ & $22.11^{\mathrm{c}}$ \\
IT99K-1245 & $2.00^{\mathrm{a}}$ & $4.78^{\mathrm{b}}$ & $10.89^{\mathrm{cd}}$ & $18.56^{\mathrm{b}}$ & $27.44^{\mathrm{a}}$ & $28.78^{\mathrm{a}}$ \\
Sed & 0.14 & 0.32 & 0.62 & 0.85 & 1.08 & 1.25 \\
\hline
\end{tabular}

*Mean values with the same letter along the same row are not significantly different, $\mathrm{p} \leq 0.05$

Table 7: Mean square from the analyses of variance for branch per plant

\begin{tabular}{cccccccc}
\hline \multicolumn{7}{c}{ Plant height (weeks after planting (WAS) } \\
\hline SV & Df & 2 WAS & 3 WAS & 4 WAS & 5 WAS & 6 WAS & 7 WAS \\
Density & 2 & $0.56 \mathrm{~ns}$ & & $0.09 \mathrm{~ns}$ & $0 \mathrm{~ns}$ & $0 \mathrm{~ns}$ & $0 \mathrm{~ns}$ \\
Error (a) & 4 & 0.29 & 0.73 & 0.02 & 0 & 0 & 0 \\
Variety & 4 & $0.14 \mathrm{~ns}$ & $0.37 \mathrm{~ns}$ & $0.09 * *$ & $0 \mathrm{~ns}$ & $0 \mathrm{~ns}$ & $0 \mathrm{~ns}$ \\
Density x variety & 8 & $0.19 \mathrm{~ns}$ & $0.97 \mathrm{~ns}$ & $0.09^{* *}$ & $0 \mathrm{~ns}$ & $0 \mathrm{~ns}$ & $0 \mathrm{~ns}$ \\
& & & & & & & \\
Error (b) & 24 & 0.33 & 0.88 & 0.02 & 0 & 0 & 0 \\
\hline${ }^{* * * * * * *}$ Significant F test at $0.05,0.01$ and 0.001 levels of probability respectively, "ns" not significant
\end{tabular}

Table 8: Effect of population density and variety on number of branches per plant

\begin{tabular}{ccccccc}
\hline Treatment & 2 WAS & 3 WAS & 4 WAS & $\mathbf{5 ~ W A S}$ & $\mathbf{6}$ WAS & $\mathbf{7 ~ W A S}$ \\
\hline 88,000 & $0.60^{\mathrm{a}}$ & $2.20^{\mathrm{b}}$ & $4.00^{\mathrm{a}}$ & $4.00^{\mathrm{a}}$ & $4.00^{\mathrm{a}}$ & $4.00^{\mathrm{a}}$ \\
44,000 & $0.27^{\mathrm{a}}$ & $2.87^{\mathrm{a}}$ & $3.87^{\mathrm{b}}$ & $4.00^{\mathrm{a}}$ & $4.00^{\mathrm{a}}$ & $4.00^{\mathrm{a}}$ \\
29,000 & $0.27^{\mathrm{a}}$ & $2.13^{\mathrm{b}}$ & $4.00^{\mathrm{a}}$ & $4.00^{\mathrm{a}}$ & $4.00^{\mathrm{a}}$ & $4.00^{\mathrm{a}}$ \\
Sed & 0.20 & 0.31 & 0.05 & 0.00 & 0.00 & 0.00 \\
Variety & & & & & \\
IT97K-461-4 & $0.56^{\mathrm{a}}$ & $2.44^{\mathrm{a}}$ & $3.78^{\mathrm{b}}$ & $4.00^{\mathrm{a}}$ & $4.00^{\mathrm{a}}$ & $4.00^{\mathrm{a}}$ \\
IT97-568-18 & $0.33^{\mathrm{a}}$ & $2.33^{\mathrm{a}}$ & $4.00^{\mathrm{a}}$ & $4.00^{\mathrm{a}}$ & $4.00^{\mathrm{a}}$ & $4.00^{\mathrm{a}}$ \\
IT98K-131-2 & $0.22^{\mathrm{a}}$ & $2.44^{\mathrm{a}}$ & $4.00^{\mathrm{a}}$ & $4.00^{\mathrm{a}}$ & $4.00^{\mathrm{a}}$ & $4.00^{\mathrm{a}}$ \\
IT99K-1060 & $0.33^{\mathrm{a}}$ & $2.67^{\mathrm{a}}$ & $4.00^{\mathrm{a}}$ & $4.00^{\mathrm{a}}$ & $4.00^{\mathrm{a}}$ & $4.00^{\mathrm{a}}$ \\
IT99K-1245 & $0.44^{\mathrm{a}}$ & $2.11^{\mathrm{a}}$ & $4.00^{\mathrm{a}}$ & $4.00^{\mathrm{a}}$ & $4.00^{\mathrm{a}}$ & $4.00^{\mathrm{a}}$ \\
Sed & 0.27 & 0.43 & 0.07 & 0.00 & 0.00 & 0.00 \\
\hline
\end{tabular}

*Mean values with the same letter along the same row are not significantly different, $\mathrm{p} \leq 0.05$ 
Table 9: means square from the analyses of variance for dry matter per plant

\begin{tabular}{ccc}
\hline SV & Df & DM \\
\hline Density & 2 & $10.64 \mathrm{~ns}$ \\
Error (a) & 4 & 4.40 \\
Variety & 4 & $399.84^{* * *}$ \\
Density x variety & 8 & $2.01 \mathrm{~ns}$ \\
Error (b) & 24 & 0.92
\end{tabular}

$*, * * ; * *$ ** Significant $\mathrm{F}$ test at $0.05,0.01$ and 0.001 levels of probability respectively, "ns" not significant

Table 10: Effects of population density and variety on dry matter per plant

\begin{tabular}{cc}
\hline Treatment & DM \\
\hline 88,000 & $25.13^{\mathrm{a}}$ \\
44,000 & $23.60^{\mathrm{a}}$ \\
29,000 & $23.75^{\mathrm{a}}$ \\
Sed & 0.766 \\
Variety & \\
IT97K-461-4 & $23.52^{\mathrm{c}}$ \\
IT97-568-18 & $33.60^{\mathrm{a}}$ \\
IT98K-131-2 & $15.33^{\mathrm{d}}$ \\
IT99K-1060 & $23.33^{\mathrm{c}}$ \\
IT99K-1245 & $25.00^{\mathrm{b}}$ \\
Sed & 0.46
\end{tabular}

*Mean values with the same letter along the same row are not significantly different, $\mathrm{p} \leq 0.05$

Means having the same letter in a column are not significantly different by DMRT $(\mathrm{p}<0.05)$

Table 11: Mean square from the analyses of variance for flower production

\begin{tabular}{|c|c|c|c|c|c|c|c|c|c|c|c|c|c|c|c|c|}
\hline SV & $\begin{array}{l}d \\
f\end{array}$ & 1 & 2 & 3 & 4 & 5 & 6 & 7 & 8 & 9 & 10 & 11 & 12 & 13 & 14 & 15 \\
\hline $\begin{array}{l}\text { Dens } \\
\text { ity }\end{array}$ & 2 & $\begin{array}{l}11.82 \\
\mathrm{~ns}\end{array}$ & $\begin{array}{l}15.26 \\
\mathrm{~ns}\end{array}$ & $\begin{array}{l}20.69 \\
* *\end{array}$ & $\begin{array}{l}26.6 * \\
*\end{array}$ & $\begin{array}{l}4.95 n \\
\mathrm{~s}\end{array}$ & $\begin{array}{l}4.42 \\
* *\end{array}$ & $\begin{array}{l}6.82 \\
\text { ns }\end{array}$ & $\begin{array}{l}0.42 n \\
s\end{array}$ & $\begin{array}{l}0.96 \\
\text { ns }\end{array}$ & 0.36 & $\begin{array}{l}0.00 \\
\mathrm{~ns}\end{array}$ & 1.42 & $\begin{array}{l}5.07 \\
\mathrm{~ns}\end{array}$ & $\begin{array}{l}2.49 \\
\mathrm{~ns}\end{array}$ & $\begin{array}{l}0.87 \\
\mathrm{~ns}\end{array}$ \\
\hline $\begin{array}{l}\text { Error } \\
\text { (a) }\end{array}$ & 4 & 1.92 & 2.57 & 1.32 & 1.87 & 0.86 & 0.55 & 2.29 & 1.16 & 1.39 & 1.79 & 0.40 & 1.06 & 1.97 & 0.75 & 1.43 \\
\hline $\begin{array}{l}\text { Vari } \\
\text { ety }\end{array}$ & 4 & $\begin{array}{l}60.33 \\
* * *\end{array}$ & $\begin{array}{l}59.86 \\
* * *\end{array}$ & $\begin{array}{l}63.61 \\
* * *\end{array}$ & $\begin{array}{l}34.37 \\
* * *\end{array}$ & $\begin{array}{l}16.91 \\
* * *\end{array}$ & $\begin{array}{l}4.03 \\
* *\end{array}$ & $\begin{array}{l}0.81 \\
\mathrm{~ns}\end{array}$ & $\begin{array}{l}0.86 n \\
s\end{array}$ & $\begin{array}{l}2.37 \\
\mathrm{~ns}\end{array}$ & 9.86 & $\begin{array}{l}5.57 \\
* *\end{array}$ & $\begin{array}{l}3.81 \\
* *\end{array}$ & $\begin{array}{l}3.26 \\
* *\end{array}$ & $\begin{array}{l}2.36 \\
\mathrm{~ns}\end{array}$ & $\begin{array}{l}0.24 \\
\mathrm{~ns}\end{array}$ \\
\hline $\begin{array}{l}\text { Dens } \\
\text { ity } \mathrm{x} \\
\text { varie } \\
\text { ty }\end{array}$ & 8 & $\begin{array}{l}7.93 * \\
* *\end{array}$ & $\begin{array}{l}1.49 n \\
s\end{array}$ & $\begin{array}{l}3.07 * \\
*\end{array}$ & $\begin{array}{l}3.43 n \\
s\end{array}$ & $\begin{array}{l}3.51 * \\
*\end{array}$ & $\begin{array}{l}1.28 \\
\mathrm{~ns}\end{array}$ & $\begin{array}{l}2.46 \\
\mathrm{~ns}\end{array}$ & $\begin{array}{l}3.172 \\
* * *\end{array}$ & $\begin{array}{l}2.57 \\
* *\end{array}$ & $\begin{array}{l}3.44 \\
* * *\end{array}$ & $\begin{array}{l}2.52 \\
\mathrm{~ns}\end{array}$ & $\begin{array}{l}1.97 \\
* *\end{array}$ & $\begin{array}{l}1.37 \\
\mathrm{~ns}\end{array}$ & $\begin{array}{l}1.74 \\
* *\end{array}$ & $\begin{array}{l}1.06 \\
\mathrm{~ns}\end{array}$ \\
\hline $\begin{array}{l}\text { Error } \\
\text { (b) }\end{array}$ & $\begin{array}{l}2 \\
4\end{array}$ & 0.71 & 1.56 & 0.94 & 1.61 & 1.18 & 0.73 & 1.11 & 0.51 & 0.60 & 0.89 & $\begin{array}{l}1.09 \\
8\end{array}$ & 0.80 & 0.83 & 0.54 & 0.66 \\
\hline
\end{tabular}


Table 12: Effects of population density and variety on flower production

\begin{tabular}{|c|c|c|c|c|c|c|c|c|c|c|c|c|c|c|c|}
\hline Treatment & 1 & 2 & 3 & 4 & 5 & 6 & 7 & 8 & 9 & 10 & 11 & 12 & 13 & 14 & 15 \\
\hline 88,000 & $6.27^{\mathrm{b}}$ & $6.67^{\mathrm{b}}$ & $6.87^{b}$ & $7.40^{\mathrm{b}}$ & $6.13^{\mathrm{b}}$ & $5.47^{\mathrm{b}}$ & $5.20^{\mathrm{b}}$ & $5.40^{\mathrm{a}}$ & $4.67^{\mathrm{a}}$ & $4.40^{\mathrm{a}}$ & $3.53^{\mathrm{a}}$ & $2.27^{\mathrm{a}}$ & $1.40^{\mathrm{b}}$ & $1.20^{\mathrm{b}}$ & $0.53^{\mathrm{a}}$ \\
\hline 44,000 & $7.47^{\mathrm{a}}$ & $8.13^{\mathrm{a}}$ & $8.93^{\mathrm{a}}$ & $9.60^{\mathrm{a}}$ & $7.27^{\mathrm{a}}$ & $6.47^{\mathrm{b}}$ & $6.40^{\mathrm{a}}$ & $5.60^{\mathrm{a}}$ & $5.13^{\mathrm{a}}$ & $4.40^{\mathrm{a}}$ & $3.53^{\mathrm{a}}$ & $2.80^{\mathrm{a}}$ & $2.33^{\mathrm{b}}$ & $2.00^{\mathrm{a}}$ & $1.00^{\mathrm{a}}$ \\
\hline 29,000 & $8.00^{\mathrm{a}}$ & $9.60^{\mathrm{a}}$ & $8.87^{\mathrm{a}}$ & $7.20^{\mathrm{b}}$ & $6.53^{\text {ba }}$ & $5.60^{\mathrm{b}}$ & $5.27^{\mathrm{b}}$ & $5.73^{\mathrm{a}}$ & $5.07^{\mathrm{a}}$ & $4.14^{\mathrm{a}}$ & $3.53^{\mathrm{a}}$ & $2.80^{\mathrm{a}}$ & $2.47^{\mathrm{a}}$ & $1.73^{\mathrm{ab}}$ & $0.87^{\mathrm{a}}$ \\
\hline Sed & 0.51 & 0.59 & 0.42 & 0.50 & 0.34 & 0.27 & 0,55 & 0.40 & 0.43 & 0.49 & 0.23 & 0.38 & 0.51 & 0.32 & 0.43 \\
\hline \multicolumn{16}{|l|}{ Variety } \\
\hline $\begin{array}{l}\text { IT97K- } \\
461-4\end{array}$ & $9.56^{\mathrm{a}}$ & $10.78^{\mathrm{a}}$ & $11.78^{\mathrm{a}}$ & $10.11^{\mathrm{a}}$ & $8.00^{\mathrm{a}}$ & $6.22^{\mathrm{ab}}$ & $5.67^{\mathrm{a}}$ & $5.67^{\mathrm{ab}}$ & $5.22^{\mathrm{ab}}$ & $5.56^{\mathrm{a}}$ & $4.67^{\mathrm{a}}$ & $2.89^{\mathrm{a}}$ & $2.33^{\mathrm{a}}$ & $2.33^{\mathrm{a}}$ & $0.89^{\mathrm{a}}$ \\
\hline $\begin{array}{l}\text { IT97-568- } \\
18\end{array}$ & $7.44^{\mathrm{b}}$ & $5.78^{\mathrm{c}}$ & $6.67^{\mathrm{c}}$ & $6.78^{\mathrm{b}}$ & $6.67^{\mathrm{b}}$ & $6.56^{\mathrm{a}}$ & $5.44^{\mathrm{a}}$ & $5.11^{\mathrm{b}}$ & $4.67^{\mathrm{c}}$ & $4.56^{\mathrm{b}}$ & $3.78^{\mathrm{ab}}$ & $3.00^{\mathrm{a}}$ & $2.33^{\mathrm{a}}$ & $1.67^{\mathrm{ab}}$ & $0.78^{\mathrm{a}}$ \\
\hline $\begin{array}{l}\text { IT98K- } \\
131-2\end{array}$ & $2.89 \mathrm{c}$ & $4.56^{\mathrm{d}}$ & $4.78^{\mathrm{d}}$ & $5.33^{\mathrm{c}}$ & $4.44^{\mathrm{c}}$ & $4.89^{\mathrm{c}}$ & $5.22^{\mathrm{a}}$ & $5.78^{\mathrm{ab}}$ & $4.33^{\mathrm{c}}$ & $2.89^{\mathrm{c}}$ & $2.56^{\mathrm{c}}$ & $1.11^{\mathrm{b}}$ & $1.00^{\mathrm{b}}$ & $1.00^{\mathrm{b}}$ & $0.56^{\mathrm{a}}$ \\
\hline $\begin{array}{l}\text { IT99K- } \\
1060\end{array}$ & $8.78 \mathrm{a}$ & $9.44^{\mathrm{b}}$ & $9.33^{\mathrm{b}}$ & $9.11^{\mathrm{a}}$ & $7.56^{\mathrm{ab}}$ & $6.11^{\mathrm{ab}}$ & $6.00^{\mathrm{a}}$ & $5.89^{\mathrm{a}}$ & $5.67^{\mathrm{c}}$ & $4.89^{\mathrm{ab}}$ & $3.56^{\mathrm{b}}$ & $3.33^{\mathrm{a}}$ & $2.44^{\mathrm{a}}$ & $1.89^{\mathrm{a}}$ & $1.00^{\mathrm{a}}$ \\
\hline $\begin{array}{l}\text { IT99K- } \\
1245\end{array}$ & $7.56^{\mathrm{b}}$ & $8.44^{\mathrm{b}}$ & $8.56^{\mathrm{b}}$ & $9.00^{\mathrm{a}}$ & $6.56^{\mathrm{b}}$ & $5.44^{\mathrm{bc}}$ & $5.78^{\mathrm{a}}$ & $5.44^{\mathrm{ab}}$ & $4.89^{c}$ & $3.67^{\mathrm{bc}}$ & $3.11^{\mathrm{bc}}$ & $2.78^{\mathrm{a}}$ & $2.22^{\mathrm{a}}$ & $1.33^{\mathrm{b}}$ & $0.78^{\mathrm{a}}$ \\
\hline Sed & 0.40 & 0.50 & 0.46 & 0.60 & 0.51 & 0.4 & 0.50 & 0.33 & 0.37 & 0.44 & 0.49 & 0.43 & 0.43 & 0.34 & 0.38 \\
\hline
\end{tabular}

*Mean values with the same letter along the same row are not significantly different, $\mathrm{p} \leq 0.05$

Table 13: Mean square from the analyses of variance for flower production

\begin{tabular}{|c|c|c|c|c|c|c|c|}
\hline SV & $\mathrm{df}$ & $\mathrm{HI}$ & SP & Seed pod & Pods & $\begin{array}{c}\text { Seed } \\
\text { weight }\end{array}$ & $\begin{array}{c}\text { Yield } \\
(\mathrm{Kg} / \mathrm{ha})\end{array}$ \\
\hline Density & 2 & $0.01 \mathrm{~ns}$ & $42.68 \mathrm{~ns}$ & $6.00 * * *$ & $47.76 \mathrm{~ns}$ & $0.01 \mathrm{~ns}$ & $170336 \mathrm{~ns}$ \\
\hline Error (a) & 4 & 0.01 & 45.53 & 0.49 & 13.29 & 0.01 & 119326 \\
\hline Variety & 4 & $0.02 \mathrm{~ns}$ & $97.42 \mathrm{~ns}$ & $6.70 * * *$ & $24.58 \mathrm{~ns}$ & $4.01 * * *$ & 76916ns \\
\hline $\begin{array}{c}\text { Density } \mathrm{x} \\
\text { variety }\end{array}$ & 8 & $0.01 \mathrm{~ns}$ & $125.30 \mathrm{a}$ & $1.23 \mathrm{~ns}$ & $40.06 * *$ & $0.01 \mathrm{~ns}$ & $27656 \mathrm{~ns}$ \\
\hline Error (b) & 24 & 0.01 & 47.89 & 0.84 & 10.97 & 10.97 & 43037 \\
\hline
\end{tabular}




\section{Discussion}

Population density of 29,000 plants/ha resulted in taller plant from other plant population densities. This agrees with Kamara et al. (2003) who stated Page | 4098 that increase in number of plant per hectare was associated with increase in plant height. This short plant height to increase in population density can be attributed to increase in moisture stress (Sing 2007) and competition for limited available nutrient. This means that the greater the plant density the lower the plant highest. The leaf production increased with increased in plant population density especially at 4WAP-7WAP with 88,000 plants/ha having the highest lead production. This observation agrees with Xiaolei and Zhifeng (2002) who stated that widely spread plants at low density, never completely cover the ground with their leaves and radiation interception is less efficient that at high population. The reason is that the increased competition at high density result in excessive leaf growth at the expense of the roots. The response on dry matter production in this study agreed with the observation of Purcell et al (2002) that dense sowing increased dry matter weight. The result from this research work showed that 88,000 plants/aha gives the highest dry matter weight which in turn gives the highest yield as compared to other densities.

Flower production was positively affected by population density with 88.000 plants/ha gave the highest flower production. This showed that dense sowing gave more flower production. This statement disagrees with (Xiaolei and Zhifeng, 2002) who stated that higher plant density decreased flower formation and increased abortion of reproductive structure due to plant competition for space, light and other growth factors. Results of this study with a significant decrease in pod formation with increased population density disagree with Purcell et al. (2002) who stated that with decreasing plant population density, there were increase in the number of pods per stem and number of seed per meter square $\left(\mathrm{m}^{2}\right)$. This is because the resources needed for the pod formation are less competed for at low densities.

In this study, number of seeds per pod increased with decreasing population density. This is in agreement with Chiezey (1990) who reported that higher density plant lead to decreased yield of individual plants. This is attributed to lesser competition for light, moisture and nutrient. The result of rain yield response to the effect of population densities showed that as population level increases, grain yield increases. This agrees with the results of Ismail and Hall (2000) who stated that higher density planting resulted in increased yield per unit area. Also it agrees with Kamara et al., 2014 who reported that yield increased with increasing number of plant per stand although individual plants yield was reduced because the lower half of the stem produced fewer pod. The best spatial distribution and density and plant population are those which maximize yield hence a population of 88,000 plants/ha was revealed to be the best for the selected specie of cowpea in this study.

\section{Conclusion}

From the experiment, it could be observed that population of 88,000 plants/ha gave the highest yield and this can be recommended for farmers' usage. Any farmer that is interested in any of cowpea varieties in Southern Guinea Savannah should go for IT97K-568-18 because it gives highest seed per pod and yield.

\section{Competing interests}

The authors declare no conflict of interest.

\section{References}

Afolabi M. S, Lamidi W. A. and Akoroda M. O. (2020) Effects of Rainfall Variability on Missing Stands and Yield in Sweet Potato (Ipomoea Batatas (L.) Lam.) Production. Published by Journal of Agricultural 
Engineering and Technology (JAET) 25(1): 95-105. (Journal of Nigerian Institution of Agricultural Engineers (NIAE)

Ajeigbe, H. A., Singh, B. B. and Emebehe, A. M. (2008). Field evaluation of improved cowpea lines for resistance to bacterial blight, virus and Striga under natural infestation in the West African savannas. African Journal of Biotechnology 7:35633568 .

Ajeigbe, H., Singh, B., Ezeaku, I. and Adeosun, J. (2010). On-farm evaluation of improved cowpea-cereals cropping systems for croplivestock farmers: Cereals-cowpea systems in Sudan savanna zone of Nigeria. African Journal of Agricultural Research 5:22972304.

Anonymous, (2008). Cowpea; Abuja securities and commodity exchange plc. http://www.abujacomx.com

Brader, I. (2002). Forward page VI in challenges and opportunities for enhancing sustainable cowpea production, edited by Fatakun, C.A., Tarawali, S. A, Singh, B.B., Kormowa, P.M. and Tamo, M. IITA, Ibadan, Nigeria.

Bremner JM 1996 Total Nitrogen. In: Sparks, D. L. (ed). Methods of Soil Analysis. Part 3. Chemical Methods. SSSA and ASA. Madison, Wisconsin, USA. 1123-1184.

Chiezey U. F. (1990) Effect of phosphorus and plant density on the yield components of soybean, tropical Science. 321: 361-368.

Dugje, I. Y., Omoigui, L. O., Ekeleme, F., Kamara, A. Y. and Ajeigbe, H. A. (2009).Farmers' guide to cowpea production in West Africa.IITA Ibadan, Nigeria.20p. Egli, D. B. (1988). Plant density and soybean yield. Crop Science 28:977-981.

Ismail, A. M. and Hall, A. E. (2000).Semi-dwarf and standard-height cowpea responses to row spacing in different environments. Crop Science 40:1618-1623.

Kamara, A. Y., Menkir, A., Badu-Apraku, B. and Ibikunle, O. (2003). The influence of drought stress on growth, yield and yield components of selected maize genotypes. Journal of Agricultural Science 141:43-50.

Kamara, A. Y., Sylvester, U. E., Tofa, A. I. and Steve, B. (2014).Agronomic response of soybean to plant density in the Guinea savannas of Nigeria. Agronomy Journal 106:1051-1059.

Kuo, S. (1996). Phosphorus. In: Sparks, D. L. (ed). Methods of Soil Analysis. Part 3. Chemical Methods. SSSA and ASA. Madison, WI. 869-920.

Muleba, N., C. Dabire, J.B such, Drabo, I. and Ovedraogo, IT. (1997). Technologies for cowpea production based on genetic and environment manipulation in the semi-arid tropics.

Nelson, D. W., Sommers L. E. (1996). Total carbon and organic matter. In: Bartels J.M. (ed) Methods of soil analysis: Part 3, Chemical methods, $3^{\text {rd }}$ edn. ASAand SSSA, Madison, W. I., Book series no 5, 9611010 .

Onyibe, J.E., Kamara, A.Y and Omoigui, L.O (2006). Guide to cowpea production in Borno state, Nigeria. Promoting sustainable Agriculture in Borno state (PROSAB). Ibadan, Nigeria. 36pp

Okalabo; J. R, Gathua. K. W, Woomer P. L (2002). Laboratory methods of soil and plant analysis. A working manual, $2^{\text {nd }}$ 
edition. Sacred African, Africa, Nairobi, Kenya.

Purcell, L. C., Ball, R. A., Reaper, J. D. and Vories, E. D. (2002). Radiation use Page | 4100 efficiency and biomass production in soybean at different plant density densities. Crop Science 42:172-177.

Singh, B.B, (2007). Potential and constraits of improved cowpea varieties in increasing the productivity of cowpea cereal system in dry savanna of west Africa pages 14-26 in Majiwa, P., Odera, M., Muchiri, N., Omanya, G. and Werehire, P. A plan to apply technology in the improvement of cowpea productivity and utilization for the benefit of farmers and consumers in Africa: proceeding of cowpea stakeholders workshop. Nairobi Kenya: African Agricultural Technology foundation.

Thomas, G.W. (1996). Soil pH and soil acidity. In: Sparks, D. L. (ed). Methods of Soil Analysis. Part 3. Chemical Methods. SSSA and ASA. Madison, WI. 475-490.

Vogl, C. R., and B. Vogl-Lukasser. 2004. Tradition, dynamics and sustainability of plant species composition and management in homegardens on organic and non-organic small-scale farms in Alpine Eastern Tyrol, Austria. Journal for Biological Agriculture and Horticulture 21:349-366.

Watson, J. W., and P. B. Eyzaguirre, eds. 2002. Proceedings of the Second International Home Gardens Workshop: Home gardens and in situ conservation of plant genetic resources in farming systems, July 17-19, 2001, Witzenhausen, Federal Republic of Germany. International Plant Genetic Resources Institute, Rome.

Xiaolei,S. andZhifeng,W.(2002).The optimal leaf area index for cucumber photosynthesis and production in plastic green house. Acta Horticulturae 633. DOI:10.17660/ActaH ortic.2004.633.19 